\title{
Cellulitis: Treatment failure or failure to define treatment failure?
}

\section{Nimai Etheridge \& Gerben Keijzers}

Dear Editor,

Cellulitis is a common ED diagnosis and antibiotics are part of routine management. Each year, almost 60000 people are hospitalised for treatment of cellulitis in Australia, of which an estimated $11 \%$ were deemed preventable. 1

Appropriate antibiotic selection involves the right antibiotic type, dosage and route of administration. Multiple randomised controlled trials and a Cochrane review2 suggest oral antibiotics are non-inferior to i.v. administration for most patients. We have previously highlighted the need to review unnecessary i.v. antibiotics use in more detail.3

Although i.v. administration is recommended for patients with sepsis or poor absorption (such as persistent vomiting or diarrhoea), many patients not meeting these criteria still receive i.v. antibiotics in an ED short stay setting. This is supported by a review of $\sim 1800$ patients who presented to our ED and received a diagnosis of cellulitis. Of these, approximately one-third were discharged home, one-third admitted to a hospital ward and one-third to the ED short stay ward. These short stay patients almost exclusively received a short course i.v. antibiotics with discharge the next day.

A fundamental problem in the cellulitis literature is the concept of 'treatment failure' after starting with oral antibiotics. Many descriptive studies define treatment failure based on the treating clinician's choice to change the antibiotics. This approach is inherently flawed, as it will perpetuate factors that have no bearing on actual treatment failure. If two doses of antibiotics have not led to a change in symptoms and the patient states the 'treatment is not working', this requires patient education and management of expectations, rather than changing the agent or route of administration. Clinicians often use parameters such as raised inflammatory markers, ongoing redness, isolated fever despite normal vital signs, 3 to change from oral to i.v. administration. None of these variables are supported by high-level evidence and likely perpetuate the current, flawed, concept of treatment failure. Given that cellulitis is a paucibacillary infection, with small amounts of bacteria which trigger an inflammatory state, ongoing symptoms after commencing appropriate oral 
antibiotics are explained by the associated inflammatory response. This implies adjuvant antiinflammatories may be more beneficial. 4

The current lack of consensus on the definition of treatment failure leads to significant practice variation. National prescribing guidelines such as the eTG recommend a switch to i.v. therapy if a patient is not responding to treatment after $48 \mathrm{~h}, 5$ but fail to define what constitutes a lack of response. To be able to progress appropriate antimicrobial choices in cellulitis, an objective definition of 'treatment failure' is required. To achieve such as definition, we need to better understand the natural course of cellulitis as part of current practice.

To this end, we have designed a cohort study where we will follow 300 consecutive patients who receive an ED diagnosis of cellulitis. All patients, regardless of antibiotic agent, dose and route of administration will have clinical features assessed during ED and hospital stay as well as at days 3, 7 and 14 after presentation to the ED. We will also assess relevant patient centred outcomes (Table 1). We anticipate that these data will provide valuable information to better understand the natural course and therapeutic response of this common condition and provide a more robust definition of 'treatment failure'.

TABLE 1. Patient centred outcomes

Time to self-adjudicated resolution

Duration of ED/hospital stay

Number of i.v. cannulation attempts

Time off work

Adverse events

Need to represent to healthcare practitioner

Received alternative diagnosis

\section{References}

1. Australian Commission on Safety and Quality in Health Care. Australian Atlas of Healthcare Variation 2017: 1.3 Cellulitis. 2017. [Cited 26 Feb 2020.] Available from URL: https://www.safetyandquality.gov.au/publications-and-resources/resourcelibrary/australian-atlas-healthcare-variation-2017-13-cellulitis-0

2. Kilburn SA, Featherstone P, Higgins B, Brindle R. Interventions for cellulitis and erysipelas. Cochrane Database Syst. Rev. 2010; CD004299. 
3. Hamill LM, Thi Y-CE, Keijzers G. Picking the low-hanging fruit: why not choose oral antibiotics for skin and soft-tissue infections in the emergency department. Emerg. Med. Australas. 2019; 31: 1119- 22.

4. Dall L, Peterson S, Simmons T, Dall A. Rapid resolution of cellulitis in patients managed with combination antibiotic and anti-inflammatory therapy. Cutis. 2005; 75: 177- 80.

5. Therapeutic Guidelines Limited. Principles of managing cellulitis and erysipelas. 2019. [Cited 14 Oct 2020.] Available from URL: https://tg-org-au /viewTopic?topicfile=cellulitiserysipelas\&guidelineName=Antibiotic\&topicNavigation=navigateTopic\#toc_d1e93 